morphological change. There into, the proportions of preautophagosome to the total area of cytoplasm in group III and group IV are remarkably decreased than that of group I, and the difference was significant $(p<0.01)$. The proportions of group II is lower than that of group I, but not significant ( $p>0.05$ ). The proportions of group IV is lower than that of group III, but there was no obvious difference between two groups ( $p>0.05)$.

Conclusion Using atorvastatin, in induction procedure, can inhibit vascular endothelial cells autophagy, which may be related to the role of atorvastatin's improvement on endothelial function. However, using atorvastatin, prior to the occurrence of induced autophagy, can not quite inhibit the occurrence of autophagy.

\section{E0035 THE STUDY OF DERIVATION FROM BONE MARROW MESENCHYMAL STEM CELLS INTO CARDIOMYOCYTE-LIKE CELLS IN VITRO VIA CARDIOTROPHIN-1}

doi:10.1136/hrt.2010.208967.35

Chaoquan Peng, Ya Gao, Peng Xiang, Ke Yang, Liyuan Zou, Xiao Wu. The Third Afiliated Hospital of Sun Yat-sen University

Objective To investigate the effects of CT-1 on differentiation of induced swine BMSCs in vitro independently or with 5-aza.

Methods BMSCs were divided into four groups : bland contro1; induced with CT-1; induced with 5-aza; induced with 5-aza combined with CT-1. After 4 weeks of induced culturing, the differentiation of cardiomyocyte induced were estimated by cTnT and a-actinin.

Result Red fluorescence staining of a-actin shows: the differentiation rate of myocardial cells of the composite group is greatest (29.900 $4.7571 \%)$; 5 -aza group is greater than CT-1 group; CT-1 group is greater than control. Red fluorescence staining of cTnT shows: the differentiation rate of myocardial cells of the composite group is greatest $(36.500 \pm 4.0927 \%) ; 5$-aza group is greater than CT-1 group; CT-1 group is greater than control.

Conclusions CT- 1 can increasing the inducing rate commbined witn 5-aza.

\section{e0036 REGULATION OF T-TYPE CA ION CHANNEL IN LYSOPHOSPHATIDYLCHOLINE-STIMULATED CARDIOMYOCYTES}

doi:10.1136/hrt.2010.208967.36

Gang Liu, Li Tian, Zhen-Guo Ji, Chao Liu, Kun-Shen Liu, Ming-Qi Zheng. First Hospital of Hebei Medical University

Objectives To study the effect of lysophosphatidylcholine (LPC) in myocardial cells in T-type calcium channel currents $\left(\mathrm{I}_{\mathrm{CaT}}\right)$, and the hypothesis that LPC accumulation in intracellular and/or interstitial space in cardiomyocytes may underlie as a mechanism for tachycardia and various arrhythmias during cardiac ischaemia.

Methods Neonatal rat cardiomyocytes from 1 to 3-day-old Wistar rats and hypertrophied ventricular myocytes from Wistar rat were prepared. A single dose of $60 \mathrm{mg} / \mathrm{Kg}$ monocrotaline was injected into the intraperitoneal cavity at the age of 8 weeks old, and right ventricular myocytes were isolated enzymatically at the age of 14 weeks from male Wister rats. In this study, whole cell patch clamp cardiac myocyte and heterologous expression of human CaV3.1 and CaV3.2 ion channel components were measured. The effect of LPC through activation of PKC isoforms mediated $\mathrm{I}_{\mathrm{CaT}}$ control mechanisms was studied.

Results (1) LPC markedly accelerated the spontaneous beating rates of neonatal rat cardiomyocytes from $42 \pm 8 \mathrm{bpm}$ in control to $64 \pm 8 \mathrm{bpm}$ after LPC application in $5 \mathrm{~min}$ at the physiological $\left[\mathrm{Ca}^{2+}\right]_{\mathrm{i}}$ condition $(\mathrm{pCa}=7.2)$. (2) In neonatal cardiomyocytes, $\mathrm{I}_{\mathrm{Ca} . \mathrm{T}}$ was significantly increased by $10 \mu \mathrm{M}$ LPC by $21.5 \%$ when $\left[\mathrm{Ca}^{2+}\right]_{\mathrm{i}}$ was high $(\mathrm{pCa}=7)$. Intracellular $\mathrm{Ca}^{2+}$-dependent augmentation of $\mathrm{I}_{\text {Ca.T }}$ by LPC was confirmed not only in neonatal cardiomyocytes but in adult ventricular myocytes from the hypertrophied heart. In this experiment, $\mathrm{I}_{\text {Ca.T }}$ was significantly increased by $10 \mu \mathrm{M}$ LPC by $23.5 \%$ when $\left[\mathrm{Ca}^{2+}\right]_{i}$ was high $(\mathrm{pCa}=7)$, although it was unchanged when $\left[\mathrm{Ca}^{2+}\right]_{\mathrm{i}}$ was low $(\mathrm{pCa}=11)$. (3) LPC exerted no effect on the Cav3.1 T-type $\mathrm{Ca}^{2+}$ channel current $\left(\mathrm{I}_{\text {Cav3.1 }}\right)$ regardless of the $\left[\mathrm{Ca}^{2+}\right]_{\mathrm{i}}$ condition at a $\mathrm{pCa}$ of 7 (solution $\mathrm{F}$ ) or at a $\mathrm{pCa}$ of 11 (solution A). In contrast, LPC upregulated the Cav3.2 T-type $\mathrm{Ca}^{2+}$ channel current ( $\mathrm{I}_{\mathrm{Cav3.2}}$ ), which was much larger at a $\mathrm{pCa}$ of 7 than that at a pCa of 11. (4) A specific PKC $\alpha$ inhibitor Ro-32-0432 completely blocked the effect of LPC on $\mathrm{I}_{\mathrm{Cav} 3.2}$. However, in the same culture condition, a specific PKC $\beta I$ inhibitor Gö 6976 (20 nM) and a specific PKC $B \mathrm{II}$ inhibitor CGP-53353 $(2 \mu \mathrm{M})$ did not modify the effect of LPC on $\mathrm{I}_{\text {Cav3.2. }}$.

Conclusion The present study indicates that intracellular signal PKC $\alpha$ activation by LPC upregulates the cardiac $\mathrm{I}_{\mathrm{Ca} . \mathrm{T}}$ in physiological or higher $\left[\mathrm{Ca}^{2}{ }^{+}\right]_{\mathrm{i}}$ condition may be a novel ischaemia-related mechanism, which may accelerate the pathophysiological cardiac automaticity and trigger tachyarrhythmias.

\section{e0037 EFFECTS OF ENALAPRIL AND IRBESARTAN ON AORTA REMODELLING AND ION PUMPS IN RENOVASCULAR HYPERTENSIVE RATS}

doi:10.1136/hrt.2010.208967.37

${ }^{1}$ Qian-Hui Shang, ${ }^{1}$ Wei Hu, ${ }^{1}$ Qian-Feng Jiang, ${ }^{2}$ Oin Wu, ${ }^{1}$ Ping Yuan. ${ }^{1}$ Institute of Clinical Medicine of Zunyi Medical College, Department of Cardiology, Affiliated Hospital of Zunyi Medical College; ${ }^{2}$ Department of Pharmacology, Zunyi Medical College, Zunyi Guizhou, China

Objective To investigate the effects of single-drug or combination therapy of enalapril and irbesartan on aorta remodelling and its mechanisms.

Methods Renovascular hypertensive rats (RHD) induced by twokidney one-clip method were treated with normal saline (mode group, $\mathrm{n}=6$ ), enalapril [10 $\mathrm{mg} /(\mathrm{kg} \mathrm{d}), \mathrm{n}=6$ ], irbesartan [50 mg/ $(\mathrm{kg} \cdot \mathrm{d}), \mathrm{n}=6$ ] and enalapril+irbesartan [5 mg/ (kg d) $+25 \mathrm{mg} /(\mathrm{kg} \mathrm{d})$ $\mathrm{n}=6]$ for 6 weeks. Six sham-operated rats were used as controls. Aortic morphology and structural changes in the media were observed by $\mathrm{HE}$ staining, immunohistochemistry and Masson staining. The content of Angiotensin II (Ang II) was measured by radioimmunoassay. The activities and mRNA levels of $\mathrm{Na}^{+}$pump and $\mathrm{Ca}^{2+}$ pump in aortic media were determined by enzyme assay and real-time PCR respectively.

Results The media area of aorta and the Ang II content were significantly increased in model group, while the activities and the mRNA levels of $\mathrm{Na}^{+}$pump and $\mathrm{Ca}^{2+}$ pump in aortic media were obviously decreased, and $\mathrm{Na}^{+}$pump and $\mathrm{Ca}^{2+}$ activities were increased in enalapril group and irbesartan group $(p<0.01)$. The Ang II content was obviously decreased in enalapril group, while increased in irbesartan group $(p<0.01)$. The mRNA levels of sodium pump $a_{1}$-subunit and plasma membrane calcium pump isoform 1 (PMCA1) in aorta smooth muscle tissue were significantly increased in enalapril group $(p<0.01)$. The amelioration of blood pressure, $\mathrm{Na}^{+}$ pump and $\mathrm{Ca}^{2+}$ pump activities, media area and thickness in combination group was significantly better than single-drug intervened group $(p<0.01)$.

Conclusion The amelioration of aorta remodelling induced by enalapril and irbesartan may be associated with the increase of $\mathrm{Na}^{+}$ pump and $\mathrm{Ca}^{2+}$ pump activities. There may be some synergistic effects on ameliorating of $\mathrm{Na}^{+}$pump and $\mathrm{Ca}^{2+}$ pump activities and aorta remodelling from combination of the two drugs. The effect of enalapril on $\mathrm{Na}^{+}$pump and $\mathrm{Ca}^{2+}$ pump activities may be mediated by increasing their mRNA expression. 